\title{
Apnea obstructiva del sueño y linfoma extraganglionar de célula T/NK tipo nasal
}

\section{Olenka Alcas-Arce ${ }^{1, *}$, Jorge Vela-Zegarra ${ }^{1}$ y Melchor Pachas-Peña ${ }^{2}$}

'Servicio de Otorrinolaringología, Departamento de Cirugía de Cabeza y Cuello; ${ }^{2}$ Departamento de Anatomía Patológica. Hospital Nacional Edgardo Rebagliati Martins, Lima, Perú

Recibido el 4 de mayo de 2017; aceptado el 8 de agosto de 2017

Disponible en Internet el 4 de diciembre de 2017

\section{PALABRAS CLAVES \\ Apnea obstructiva del sueño; \\ Linfoma \\ extraganglionar de \\ célula NK-T tipo \\ nasal; \\ Neoplasia}

\section{KEY WORDS}

Obstructive sleep apnea;

Extranodal NK-T-cell lymphoma nasal type; Neoplasms

\begin{abstract}
Resumen El linfoma extraganglionar de célula T/NK tipo nasal se presenta en varones de edado media y compromete frecuentemente el tracto aerodigestivo con síntomas iniciales inespecíficos como rinorrea, obstrucción nasal y epistaxis. Presentamos el caso de un varón de 43 años con ronquido, apneas, somnolencia y alteración de la concentración, obstrucción nasal y rinorrea; el resultado de la poligrafía cardiorrespiratoria confirmó el síndrome de apnea obstructiva del sueño (SAOS) severa. En la nasofibroscopia se observó una tumoración irregular en la nasofaringe, mientras que la tomografía además concluyó la presencia de adenopatías a nivel I, II y III. La biopsia nasofaríngea mostró un linfoma extraganglionar de células T/NK tipo nasal, continuando con quimioterapia y radioterapia. (creativecommons.org/licenses/by-nc-nd/4.0/),
\end{abstract}

Abstract The extranodal T/NK-cell nasal type occurs in middle-aged men, and often compromisos the aerodigestive tract with nonspecific initial symptoms such as rhinorrhea, nasal obstruc $=$ tion and epistaxis. We report the case of a 43-year-old male with snoring, apneas, drowsiness and impaired concentration, nasal obstruction and rhinorrhea. The cardiorespiratory polygraphy confirmed severe obstructive sleep apnea syndrome. Flexible nasofibroscopy showed an irregular tumor in nasopharynx; while the tomography also concluded with the presence of lymphadenopathy in level I, III and III. Nasopharyngeal biopsy showed extranodal T/NK-cell nasal type, continuing with chemotherapy and radiotherapy.

*E-mail para correspondencia: olenka.alcas@unmsm.edu.pe (O. Alcas-Arce) 


\section{INTRODUCCIÓN}

El linfoma extranodal T/NK tipo nasal es más frecuente en hombres de edad media y en el $80 \%$ de casos afecta al tracto aerodigestivo, incluyendo la nariz y los senos paranasales ${ }^{1}$. Los síntomas son inespecíficos e incluyen rinorrea, obstrucción nasal y epistaxis ${ }^{2}$, por lo que inicialmente estos casos son vistos en otorrinolaringología. El diagnóstico suele retrasarse por múltiples biopsias con muestra insuficiente o áreas de necrosis, y el tratamiento incluye radioterapia y quimioterapia ${ }^{2,3}$.

\section{PRESENTACIÓN DEL CASO}

Se presenta el caso de un paciente varón de 43 años con antecedente de hipertensión controlada y amigdalectomía a los 42 años (con resultado de amigdalitis crónica). Acude por ronquido, pausas respiratorias nocturnas y nicturia, y refiere haberse quedado dormido conduciendo, así como alteración de la concentración y la memoria, con una disminución del rendimiento laboral. Además presenta una obstrucción nasal bilateral, rinorrea y plenitud aural. La escala de Epworth es de 24 y en el examen físico se encuentra un índice de masa corporal de 36.89, perímetro del cuello de $52 \mathrm{~cm}$, septum nasal desviado, cornetes hipertróficos y pálidos, amígdalas de grado 0, Mallampati III y Friedman Tongue Position III. La poligrafía cardiorrespiratoria evidencia un índice de apnea-hipopnea (IAH) de 102, índice de desaturación de oxígeno del $68 \%$, saturación < $90 \%$ : $60 \%$, con diagnóstico de SAOS severa.

En la nasofibroscopia flexible se encontró una masa irregular en la nasofaringe, de aspecto friable, que obstruía las dos trompas de Eustaquio. La tomografía del cavum faríngeo reveló un engrosamiento mucoso concéntrico con burbujas aéreas dependiente de la pared posterior de la nasofaringe, que obstruía la nasofaringe y el tercio posterior de ambas fosas nasales, así como el seno maxilar izquierdo con contenido mucoso y conglomerados adenopáticos en el nivel I, II y III izquierdo menor de $4 \mathrm{~cm}$, y de menor tamaño en el nivel I y II derecho, el mayor de $2.8 \mathrm{~cm}$ (Fig. 1). Durante la evolución presentó una masa cervical sólida izquierda de $6 \times 4 \mathrm{~cm}$, dolorosa al tacto y no adherida a planos profundos.

La biopsia nasofaríngea en la sala de operaciones demostró un proceso linfoproliferativo de células $\mathrm{T}$ de alto grado de malignidad con aspecto citomorfológico de las células neoplásicas y patrón angiocéntrico que sugirió un linfoma extranganglionar de células T/NK tipo nasal. El estudio ampliado de inmunohistoquímica fue positivo para CD3, CD20, Ki67 con $80 \%$ y granzima B, y negativo para pancK, CD30 y CD56 (Fig. 2).

Se inició quimioterapia con vincristina, L-asparraginosa y prednisolona, con radioterapia asociada. Actualmente el paciente se encuentra en tratamiento con una respuesta favorable y mejoría de los síntomas nocturnos.

\section{DISCUSIÓN}

En nuestro caso, el paciente presentó síntomas iniciales de SAOS, que se caracteriza por episodios repetidos de obstrucción/colapso parcial o total de la vía aérea superior duran-

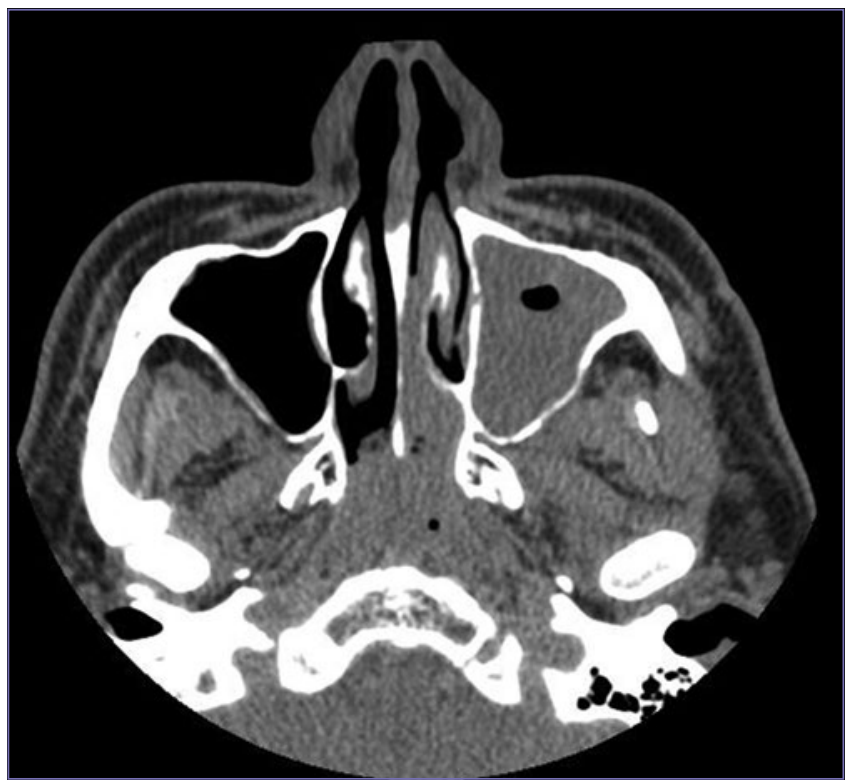

Figura 1. Tomografía espiral multicorte de la nasofaringe: corte axial con engrosamiento dependiente de la pared posterior de la nasofaringe.

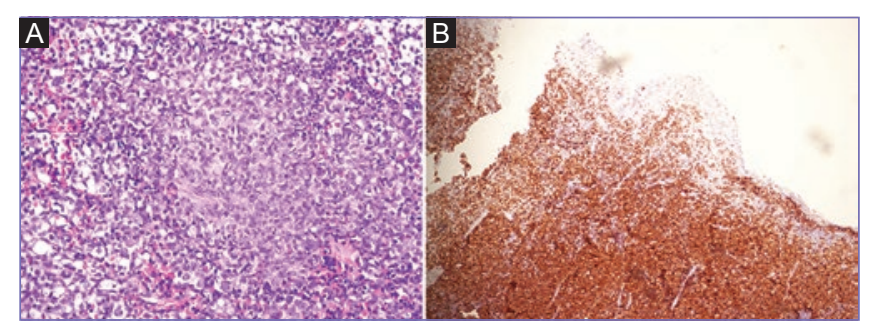

Figura 2. Anatomía patológica. A: tinción de hematoxilina-eosina: proceso linfoproliferativo con patrón angiocéntrico.

B: inmunohistoquímica ki67: positivo $80 \%$.

te la noche, con una prevalencia de entre el 5 y el $20 \%$, dependiendo de la población estudiada ${ }^{4}$. Los hombres tie nen 2-3 veces más riesgo de tener SAOS, y la sintomatologia principal es somnolencia diurna, fatiga, nicturia, alteración de la memoria y cognitiva, cefalea y disminución de la libido. El diagnóstico se realiza mediante la polisomnografía; no obstante, su elevado costo y la dificultad para su implementación han llevado a la aplicación de otros tipos de estudios del sueño como la poligrafía cardiorrespiratoria, in dicados en pacientes sin comorbilidades significativas y alta sospecha de SAOS, como fue el caso de nuestro paciente Las complicaciones asociadas a esta enfermedad son: en fermedad cardiovascular, que incluye hipertensión, enfermedad arterial coronaria e infarto cerebrovascular. También aumenta la morbilidad y mortalidad de accidentes de tránsito, diabetes tipo 2 y complicaciones postoperatorias, y la calidad de vida en general ${ }^{4}$.

Se ha postulado que la apnea obstructiva del sueño también puede causar cáncer o su progresión, posiblemente a través de hipoxia intermitente. El estudio de cohortes en Wisconsin concluye que los pacientes con $\mathrm{IAH}>30$ tienen 4.8 veces más riesgo de mortalidad por cáncer que aquéllos sin SAOS, y el porcentaje de saturación < 90\% (TSat90) está asociado significativamente a mortalidad por cáncer ${ }^{6}$. Un estudio de cohorte multicéntrico en España reportó que los pacientes con SAOS tienen mayor incidencia de cáncer, 
que aumentó 1.07 veces por cada $10 \%$ de incremento en el TSat90. Sin embargo, esto sólo era válido para pacientes varones menores de 45 años ${ }^{7}$. Otro estudio con 5,894 pacientes no encontró ninguna correlación entre síntomas diurnos y cáncer. No obstante, si se agrupaban por edades, los pacientes menores de 50 años con somnolencia diurna significativa tenían 4.09 veces más incidencia de cáncer que el grupo control. Además, la incidencia de tumores virales/inmunes (por ejemplo, leucemia y melanoma) y la de tumores relacionados con el alcohol (por ejemplo, tumores hepáticos e intestinales) en pacientes con somnolencia diurna fueron 2.73 y 4.92 veces mayores que en el grupo control ${ }^{8}$. Estos estudios demuestran una asociación entre el cáncer y el SAOS, pero no prueban causalidad.

Los posibles mecanismos por los cuales el SAOS promueve el desarrollo y progresión del cáncer no están claros. Se postula que la hipoxia intermitente seguida de reoxigenación posthipóxica produce radicales libres de oxígeno y aumenta el estrés oxidativo, lo cual daña el ADN, y se cree que es un factor importante en la tumorogénesis. Además, la hipoxia intermitente promueve la sobreexpresión del factor inducible por hipoxia, que tiene un rol importante en la angiogénesis tumoral y es altamente expresado en muchos tumores sólidos ${ }^{9,10}$.

En el presente caso, el paciente varón menor de 45 años presentaba un IAH y un TSat90 bastante elevados, que coincide con los factores de riesgo asociados a cáncer en pacientes con SAOS. Si bien la sintomatología y el examen físico inicial sugerían SAOS, la poligrafía con un IAH extremo y la nasofibroscopia hicieron sospechar una neoplasia concomitante, con el hallazgo final de linfoma extranodal T/NK tipo nasal, una entidad no tan infrecuente en nuestro medio. Este reporte resalta la necesidad de considerar el SAOS como una presentación de tumores del tracto aerodigestivo, principalmente de linfomas no Hodgkin, según los casos clínicos reportados en la literatura ${ }^{11-13}$, así como de realizar un exhaustivo plan de trabajo que incluya una nasofibroscopia y una tomografía.

\section{CONFLICTO DE INTERESES}

Los autores declaran no tener ningún conflicto de intereses.

\section{BIBLIOGRAFÍA}

1. Suzuki R. NK/T-cell lymphomas: pathobiology, prognosis and treatment paradigm. Curr Oncol Rep. 2012;14(5):395-402.

2. Miyake MM, Oliveira MV, Miyake MM, Garcia JO, Granato L. Clinical and otorhinolaryngological aspects of extranodal NK/T cell lymphoma, nasal type. Braz J Otorhinolaryngol. 2014;80(4):325-9.

3. Kwong YL. The diagnosis and management of extranodal NK/T-cell lymphoma, nasal-type and aggressive NK-cell leukemia. J Clin Exp Hematop. 2011;51(1):21-8.

4. Palamaner Subash Shantha G, Kumar AA, Cheskin LJ, Pancholy SB: Association between sleep-disordered breathing, obstructive sleep apnea, and cancer incidence: a systematic review and meta-analysis: Sleep Med. 2015;16(10):1289-94. [Internet]. Disponible en: http://www.ncbi.nlm.nih.gov/pubmed/26212231.

5. Gibson GJ. Obstructive sleep apnoea syndrome: Underestimated and undertreated. Br Med Bull. 2004;72:49-64.

6. Nieto FJ, Peppard PE, Young T, Finn L, Hla KM, Farré R. Sleep-disore dered breathing and cancer mortality: results from the Wisconsin Sleep Cohort Study. Am J Respir Crit Care Med. 2012;186(2):190-4. [Internet] Disponible en: http://www.ncbi.nlm.nih.gov/pubmed/22610391.

7. Campos-Rodriguez F, Martinez-Garcia MA, Martinez M, et al. Association between Obstructive Sleep Apnea and Cancer Incidence in a Large Multicenter Spanish Cohort. Am J Respir Crit Care Med. 2013;187(1):99-105. [Internet] Disponible en: http://www.atsjournals $\mathrm{org} / \mathrm{doi} / \mathrm{abs} / 10.1164 / \mathrm{rccm} .201209-16710 \mathrm{C}$.

8. Christensen AS, Clark A, Salo P, et al. Symptoms of Sleep Disordered Breathing and Risk of Cancer : A Prospective Cohort Study. Sleep. 2013;10(36):1429-35. [Internet] Disponible en: http://dx.ddi org/10.5665/sleep.3030.

9. Cao J, Feng J, Li L, Chen B. Obstructive sleep apnea promotes cancer development and progression: a concise review. Sleep Breath. 2015;19(2):453-7. [Internet] Disponible en: http://link.springer: $\mathrm{com} / 10.1007 / \mathrm{s} 11325-015-1126-\mathrm{x}$.

10. Gozal D, Farré R, Nieto FJ. Obstructive sleep apnea and cancer: epidemiologic links and biological plausibility. Sleep Med Rev. 2016;27:43-55. [Internet] Disponible en: http://dx.doi.org/10.1016/j.smrv.2015.05.006.

11. Gómez-Merino E, Arriero JM, Chiner E, Signes-Costa J, Marco J. Obstructive Sleep Apnea Syndrome as First Manifestation of Pharyngeal Non-Hodgkin's Lymphoma. Respiration. 2003;70(1):107-9. [Internet] Disponible en: 10.1159/000068423\nhttp://ezproxy.net.ucf.edu/lb gin?url=http: / / search.ebscohost.com/login. aspx?direct=true\&d d $\mathrm{b}=$ aph\&AN=20795575\&site $=$ ehost-live.

12. Zhu SJ, Wang QY, Zhou SH, Bao YY, Wang SQ. Obstructive sleep apnea syndrome caused by uncommon tumors of the upper aerodigestive tract. Int J Clin Exp Pathol. 2014;7(10):6686-93. [Internet] Disponible en: http://www. pubmedcentral.nih.gov/articlerender.fcgi?arti$\mathrm{d}=4230070 \&$ tool $=$ pmcentrez\&rendertype $=$ abstract.

13. Tsou YA, Cheng YK, Lin CD, Chang WC, Tsai MH. Small B cell lymphocytic lymphoma presenting as obstructive sleep apnea. World J Surg Oncol. 2004;2:26. 